\title{
Successful Pancreatic Endotherapy after Demonstration of Anatomical Variation in Pancreatic Duct by Endoscopic Ultrasound Pancreatography
}

\author{
Surinder Rana ${ }^{1}$, Ravi Sharma ${ }^{2}$, Rajesh Gupta ${ }^{3}$
}

\begin{abstract}
Aim: We aim to describe the role of accessory pancreatic duct in pancreatic endotherapy as well as role of endoscopic ultrasound (EUS) pancreatography in the demonstration of pancreatic duct anatomy.

Case description: We describe a 25-year-old male, chronic alcoholic, who presented with recurrent upper abdominal pain and investigations revealed a diagnosis of chronic calcific pancreatitis. Endoscopic retrograde pancreatography (ERP) failed as a guide wire could not be negotiated deep into pancreatic duct. EUS-guided pancreatography revealed that contrast was entering duodenum through the minor papilla via the patent accessory duct. The contrast was clearly seen exiting into the duodenum through the minor papilla orifice and successful minor papilla endotherapy could be done.

Conclusion: The patent accessory duct can provide a drainage route for endoscopic interventions via the minor papilla even in patients without pancreas divisum, and EUS pancreatography can help in delineating pancreatic duct anatomy in cases where ERP fails.

Keywords: Endosonography, Intravenous, Magnetic resonance cholangiopancreatography, Pancreas.

Journal of Postgraduate Medicine, Education and Research (2020): 10.5005/jp-journals-10028-1355
\end{abstract}

\section{INTRODUCTION}

The accessory pancreatic duct is part of pancreatic ductal anatomy and enters the duodenum through the minor papilla.' The accessory duct is usually rudimentary and its patency varies from $12 \%$ to $82 \%$ depending upon the study population as well as the method used to delineate the accessory duct. ${ }^{1,2}$ The patent accessory duct can provide a drainage route for endoscopic interventions via the minor papilla even in patients without pancreas divisum. ${ }^{3}$ In this case report, we describe a young male with painful chronic calcific pancreatitis and failed endoscopic retrograde pancreatography (ERP) and endoscopic ultrasound (EUS)-guided pancreatography helped in delineation of accessory duct and this led to successful pancreatic endotherapy.

\section{Case Description}

A 25-year-old male, chronic alcoholic, presented with recurrent upper abdominal pain of 3-month duration. Computed tomography (CT) revealed atrophic pancreas with heterogeneous attenuation and dilated pancreatic duct (PD) in body and tail (arrows; Fig. 1). Endoscopic ultrasound (EUS) revealed features of chronic pancreatitis and large ductal calculi in head of pancreas causing upstream dilatation of PD. Endoscopic retrograde pancreatography (ERP) revealed a dilated PD along with large ductal calculi in the head of pancreas (arrows; Fig. 2). The guide wire could not be negotiated deep into the PD across the large impacted ductal calculi despite repeated attempts. The minor papilla also could not be visualized and therefore the procedure was abandoned. The patient subsequently underwent an attempted EUS-guided rendezvous procedure. The dilated PD was punctured in the body of pancreas under EUS guidance and pancreatography revealed findings similar to ERP with no contrast going downstream across the ductal calculi (Fig. 3A). A tight stricture was noted distal to calculi (Fig. 3B; arrows). Repeated attempts to \begin{tabular}{l} 
1,2Department of Gastroenterology, Postgraduate Institute of Medical \\
Education and Research, Chandigarh, India \\
3Division of Surgical Gastroenterology, Department of Surgery, Post- \\
graduate Institute of Medical Education and Research, Chandigarh, India \\
Corresponding Author: Surinder Rana, Department of \\
Gastroenterology, Postgraduate Institute of Medical Education \\
and Research, Chandigarh, India, Phone: +91 172-2541044, e-mail: \\
drsurinderrana@yahoo.co.in \\
How to cite this article: Rana S, Sharma R, Gupta R, et al. Successful \\
Pancreatic Endotherapy after Demonstration of Anatomical Variation \\
in Pancreatic Duct by Endoscopic Ultrasound Pancreatography. \\
J Postgrad Med Edu Res 2020;54(2):53-55. \\
Source of support: Nil \\
Conflict of interest: None \\
\hline \hline
\end{tabular}

push the guide wire down into the duodenum across the calculi failed and therefore the rendezvous procedure was abandoned. Following the removal of the EUS endoscope, the PD anatomy was clearly demonstrated (Fig. 4) and contrast could be seen flowing into the duodenum (Fig. 4; arrows).

The contrast was seen entering the duodenum through the minor papilla via the patent accessory duct (Fig. 4; arrows). The side viewing duodenoscope was immediately introduced into the duodenum and the contrast was clearly seen exiting into the duodenum through the minor papilla orifice. The deep cannulation of the dorsal duct was achieved using a tapered cannula and a hydrophilic guide wire (Fig. 5). A 5-Fr pancreatic stent was placed through the minor papilla after minor papillotomy and bougie dilatation of the accessory duct. After the procedure, the patient had marked improvement in his symptoms and is pain free 4 weeks after successful pancreatic endotherapy.

() The Author(s). 2020 Open Access This article is distributed under the terms of the Creative Commons Attribution 4.0 International License (https://creativecommons. org/licenses/by-nc/4.0/), which permits unrestricted use, distribution, and non-commercial reproduction in any medium, provided you give appropriate credit to the original author(s) and the source, provide a link to the Creative Commons license, and indicate if changes were made. The Creative Commons Public Domain Dedication waiver (http://creativecommons.org/publicdomain/zero/1.0/) applies to the data made available in this article, unless otherwise stated. 


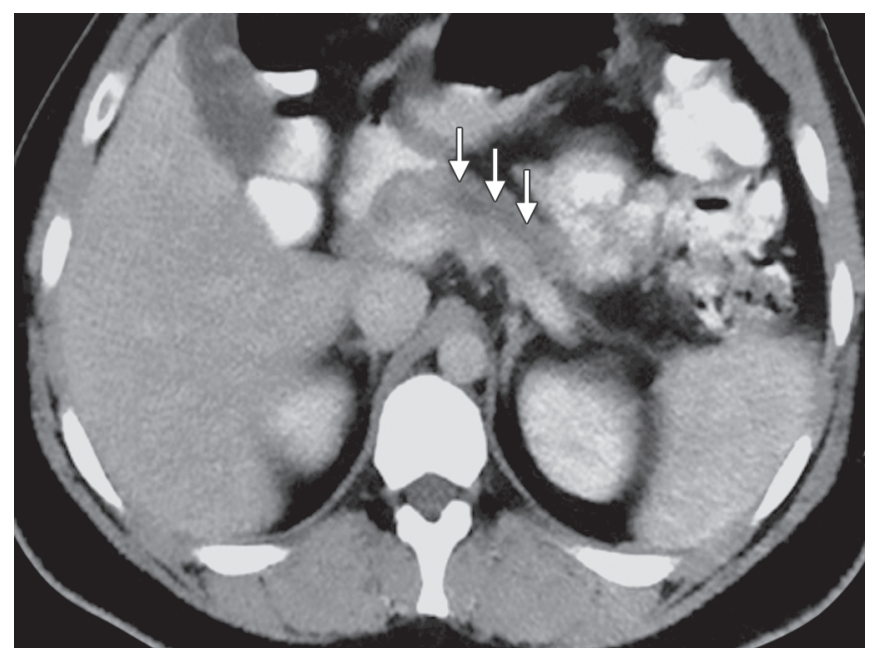

Fig. 1: Computed tomography: Atrophic pancreas with heterogeneous attenuation and dilated pancreatic duct in body and tail (arrows)

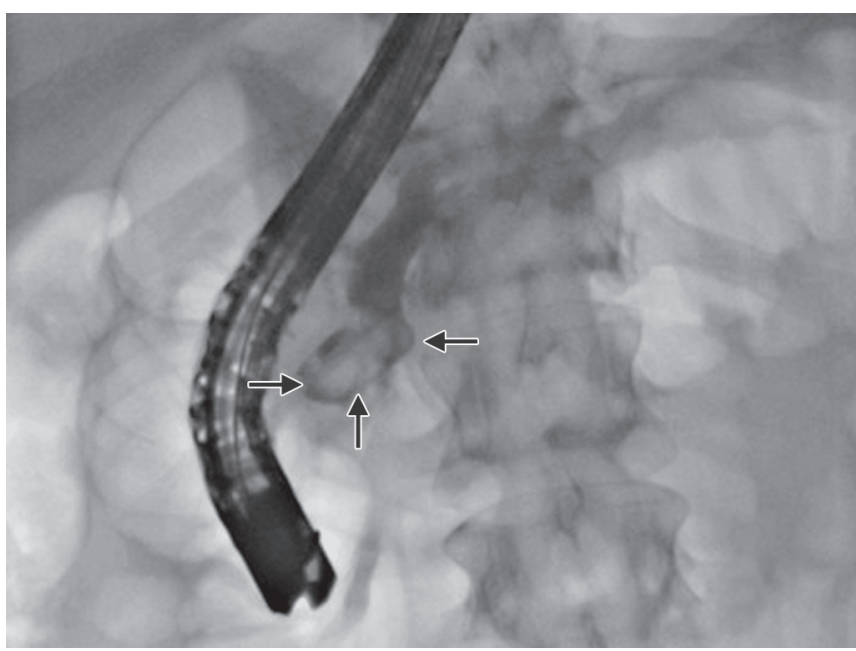

Fig. 2: Endoscopic retrograde pancreatography: Dilated pancreatic duct along with large ductal calculi in the head of pancreas (arrows)

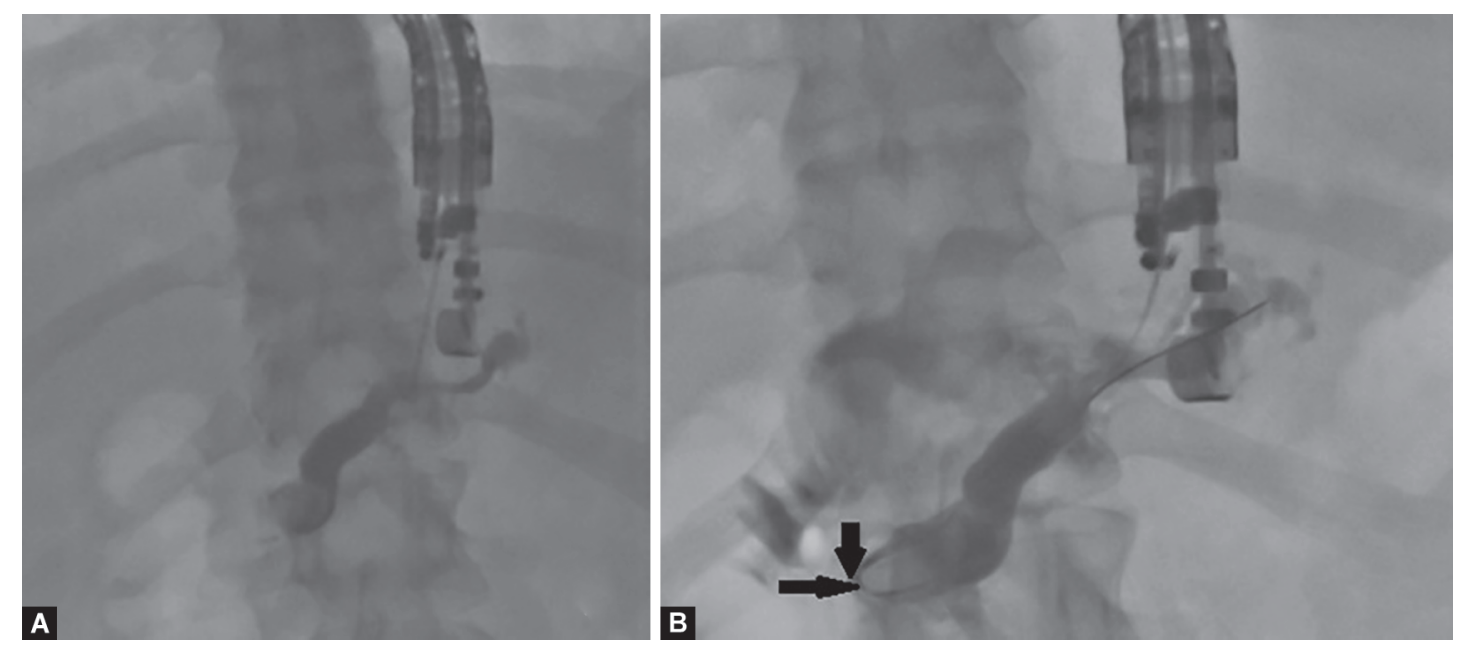

Figs $3 \mathrm{~A}$ and B: Endoscopic ultrasound pancreatography. (A) Dilated pancreatic duct along with large ductal calculi in the head of pancreas; (B) A tight stricture noted distal to calculi (arrows)

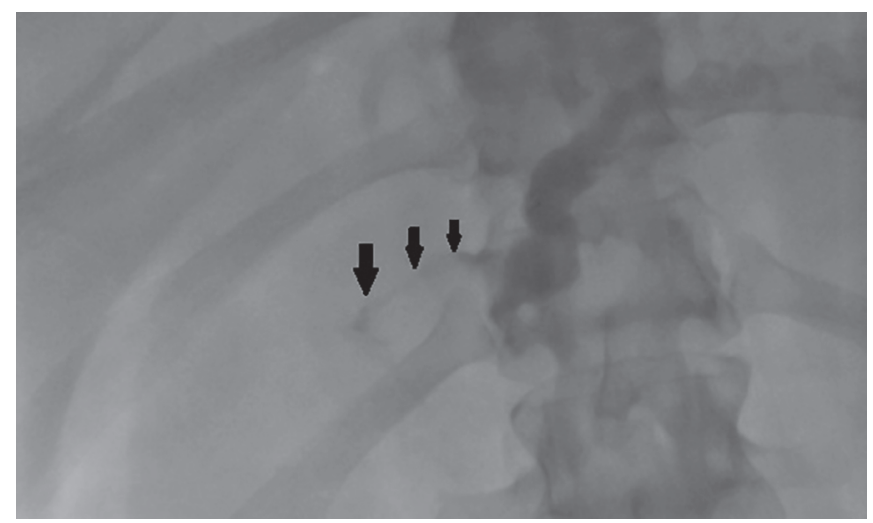

Fig. 4: Endoscopic ultrasound pancreatography: Contrast seen entering duodenum through minor papilla via the patent accessory duct (arrows)

The accessory PD enters the duodenum through the minor papilla and is the drainage duct of the dorsal pancreatic bud in the embryo. ' During embryogenesis both dorsal and ventral pancreatic

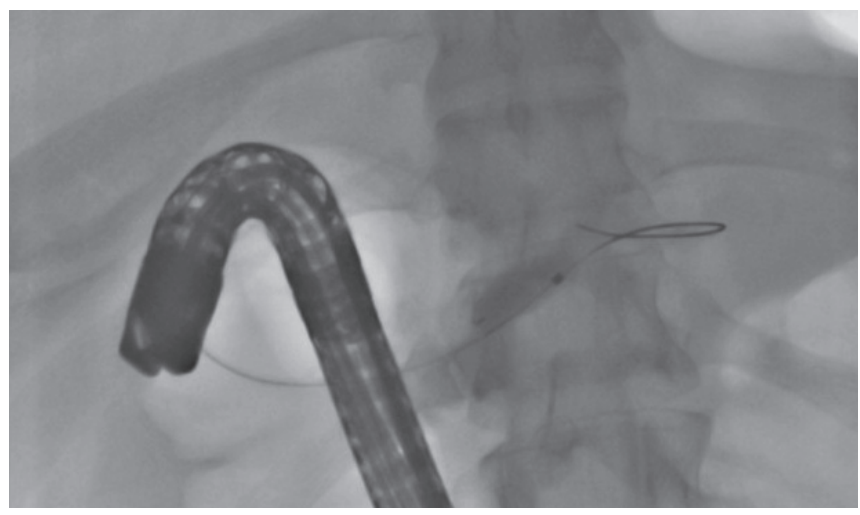

Fig. 5: Endoscopic retrograde pancreatography: Deep cannulation of dorsal duct achieved using a tapered cannula and a hydrophilic guide wire

buds fuse leading to anastomosis of dorsal and ventral ducts and the point of union of ducts usually lies between the isthmus and head of the pancreas. ${ }^{1}$ This fused duct usually becomes the main 
PD and the proximal part of the dorsal duct regresses variably to form the accessory PD., ${ }^{1,2}$ The patency of the accessory duct has been reported to vary from 12 to $82 \%$ depending upon the study population as well as the method used to delineate the accessory duct. ${ }^{1,2}$ The patent accessory duct can provide a drainage route for endoscopic interventions via the minor papilla even in patients without pancreas divisum. ${ }^{3}$ This is especially useful when it is difficult to access the main PD through the major papilla because of stricture, stone, or distortion of the course of duct despite being able to obtain pancreatogram as was in the index case.

\section{Author Contributions}

Surinder Singh Rana contributed to design, collection, and interpretation of data and drafting of manuscript. Ravi Sharma and Rajesh Gupta contributed to collection and interpretation of data.

\section{References}

1. Prasanna LC, Rajagopal KV, Thomas HR, et al. Accessory pancreatic duct patterns and their clinical implications. J Clin Diagn Res 2015;9(3):AC05-AC07. DOI: 10.7860/JCDR/2015/11539. 5660.

2. Kamisawa T, Takuma K, Tabata T, et al. Clinical implications of accessory pancreatic duct. World J Gastroenterol 2010;16(36): 4499-4503. DOI: 10.3748/wjg.v16.i36.4499.

3. Song $M H$, Kim M-H, Lee SK, et al. Endoscopic minor papilla interventions in patients without pancreas divisum. Gastrointest Endosc 2004;59(7):901-905. DOI: 10.1016/s0016-5107(04)00457-2. 